there are conflicting views regarding CVD as a major public health problem for the urban poor, including those living in slums. We examined multivariable risk prediction in a slum population and assessed the number of cardiovascular related deaths within 10 years of application of the tool.

Methods We analysed data from a cross sectional survey conducted in the Nairobi Urban Health Demographic Surveillance population (residents of two slum communities) between May 2008 and April 2009. We used the World Health Organisation/International Society of Hypertension (WHO/ISH) cardiovascular risk prediction tool to examine 10-year risk of major CVD events in a slum population. 3063 men and women aged over 40 years with complete data for variables needed for the WHO/ISH risk prediction tool were eligible for inclusion in our analysis. CVD deaths in the cohort, reported up until June 2018 in regular demographic data collection rounds, with the cause identified through verbal autopsy are also presented. Non-fatal CVD events were not captured.

Results The majority of study members (2895, 94.5\%) were predicted to have 'low' risk $(<10 \%)$ of a cardiovascular event over the next 10 years and just $51(1.7 \%)$ to have 'high' CVD risk $(\geq 20 \%)$. 91 CVD deaths were reported for the cohort up until June 2018. Of individuals classified as low risk, $74(2.6 \%)$ were identified as having died of CVD. Nine $(7.7 \%)$ of individuals classified at $10-20 \%$ risk and eight (15.9\%) classified at $>20 \%$ were identified as dying of CVD.

Discussion To the best of our knowledge this is the first study to apply a multivariable risk prediction tool to a population in a slum or informal settlement. This is a low risk population profile in comparison to results from application of multivariable risk prediction tools in other LMIC populations. This indicates that CVD may be lesser issue in slums than in other areas of LMICs cities. We found evidence that the WHO/ISH tool distinguished groups at relatively lower or higher risk of CVD events. While the absolute risk in this population is over-estimated by the tool, this may be due to limitations in our study such as lack of data on non-fatal CVD events. Our findings have implications for health service planning in similar settings.

\section{P59 HOW DO ASSOCIATIONS BETWEEN DIET QUALITY AND METABOLIC RISK VARY WITH AGE? A CROSS- SECTIONAL ANALYSIS IN A UK-REPRESENTATIVE SAMPLE}

EM Winpenny*, EMF van Sluijs, NG Forouhi. MRC Epidemiology Unit, University of Cambridge, Cambridge, UK

\subsection{6/jech-2019-SSMabstracts.210}

Background Higher diet quality shows associations with decreased risk of all-cause, cardiovascular disease, and cancer mortality in adults. To understand whether these associations also apply in younger age groups, we can study proximal metabolic risk factors: abdominal obesity, insulin resistance, hypertension and dyslipidemia. In this study we investigate how associations between diet quality and metabolic risk vary with age.

Methods We use data $(n=2105)$ from the UK-representative National Diet and Nutrition Survey (2008-2016), across three age groups: adolescents (age 11-18), young adults (age 1935), older adults (age 36-60). Four-day food diaries were processed to give an energy-adjusted diet quality index, based on the Dietary Approaches to Stop Hypertension (DASH) diet. Measures of plasma vitamin $\mathrm{C}$, beta-carotene and lutein were combined to form a fruit and vegetable $(\mathrm{F} \& \mathrm{~V})$ biomarker score. Data on the five components of metabolic syndrome (waist circumference, blood triglycerides, blood highdensity lipoprotein (HDL) cholesterol, blood pressure, fasting plasma glucose) were standardized by age, sex and ethnicity and combined to give a metabolic risk $\mathrm{z}$-score. We assessed associations of (1) standardized DASH index and (2) standardized F\&V biomarker score with metabolic risk z-score, across all ages, adjusted for potential confounders. We tested for interaction of the exposure with the three age groups, to understand moderation of effect estimates by age.

Results Adolescents and young adults showed lower selfreported diet quality $(\mathrm{p}<0.001)$, and lower $\mathrm{F} \& \mathrm{~V}$ biomarker scores $(p<0.05)$ compared to older adults. Across the whole analysis sample, both standardized DASH index (beta $=-0.15$, CI -0.22, -0.08) and standardized F\&V biomarker score (beta $=-0.33$, CI $-0.39,-0.27$ ) were associated with metabolic risk z-score. Both DASH index and F\&V biomarker score showed significant interactions with age group, with smaller associations with metabolic risk seen among adolescents and young adults compared to older adults $(p<0.05)$. Associations between F\&V biomarker score and metabolic risk remained significant across all age groups (adolescent: beta $=-0.17$, CI $-0.26,-0.07$, young adult: beta $=-0.26$, CI $0.36,-0.17$, older adult beta $=-0.39$, CI $-0.47,-0.32$ ) while associations between DASH index and metabolic risk were attenuated below significance in adolescent and young adult groups (adolescent: beta $=-0.00$, CI -0.07 , 0.08, young adult: beta $=-0.07$, CI $-0.19,0.04)$.

Conclusion Higher diet quality was associated with decreased metabolic risk, with stronger and more persistent associations seen using nutritional biomarkers, compared to self-reported dietary data. Across both diet measures, we find weaker crosssectional associations between diet quality and metabolic risk in young people compared to older populations.

\section{P60 COMPARATIVE TRENDS IN CORONARY HEART DISEASE SUBGROUP HOSPITALISATION RATES IN ENGLAND AND AUSTRALIA: A POPULATION-BASED OBSERVATIONAL STUDY, 1996-2013}

${ }^{1} \mathrm{FL}$ Wright ${ }^{*},{ }^{2} \mathrm{M}$ Greenland, ${ }^{1} \mathrm{R}$ Goldacre, ${ }^{2} \mathrm{D}$ Lopez, ${ }^{1} \mathrm{M}$ Goldacre, ${ }^{2} \mathrm{M}$ Hobbs, ${ }^{2} \mathrm{M}$ Knuiman, ${ }^{2}$ FM Sanfilippo, ${ }^{2} \mathrm{~L}$ Nedkoff. 'Unit of Health Care Epidemiology, Nuffield Department of Population Health, University of Oxford, Oxford, UK; ${ }^{2}$ School of Population and Global Health, The University of Western Australia, Perth, WA, Australia

\subsection{6/jech-2019-SSMabstracts.211}

Background Population-based coronary heart disease (CHD) studies have historically focused on myocardial infarction (MI) with limited data on trends across the spectrum of CHD. We investigated trends in hospitalisation rates for acute and chronic CHD subgroups in England and Australia from 19962013.

Methods CHD hospitalisations for 35-84 year olds were identified using the primary diagnosis in electronic hospital records from 1996-2013 for England and Australia and from the Oxford Region and Western Australia (WA). CHD subgroups identified were acute coronary syndromes (MI and unstable angina) and chronic CHD (stable angina and 'Other CHD'). We calculated age-standardised and age-specific rates, and estimated annual changes (95\% CI) from age-adjusted Poisson 
regression separately for 1996-2003 and 2004-2013 to account for non-linear trends. Regional person-linked data from the Oxford Region and WA were used to account for the effect of transfers and coronary procedure admissions on trends.

Results From 1996-2013, there were 4.9 million CHD hospitalisations in England and 2.6 million in Australia (67\% men). From 1996-2003, there was between-country variation in the direction of trends in acute coronary syndromes (ACS) and chronic CHD hospitalisation rates $(\mathrm{p}<0.001)$. During 20042013, reductions in ACS hospitalisation rates were greater than for chronic CHD hospitalisation rates in both countries, with the largest subgroup declines in unstable angina [England: men $-7.1 \% /$ year (95\% CI -7.2 to -7.0 ), women $-7.5 \%$ / year $(-7.7$ to -7.3$)$; Australia: men $-8.5 \% /$ year (-8.6 to -8.4$)$, women $-8.6 \% /$ year $(-8.8$ to -8.4$)$ ]. Age-specific trends generally reflected overall downward trends in each subgroup except for MI rates in women aged 35-54 years in 2004-2013, [England: $0 \% /$ year $(-0.5$ to +0.4$)$; Australia: $+1.9 \% /$ year $(+1.4$ to +2.4$)]$. Rates of 'Other CHD' increased in 75-84 year olds in both countries. Chronic CHD comprised half of all CHD admissions, with the majority involving angiography or revascularisation. Analysis of linked regional data found increasing MI rates in WA from 2004-2013 for men and women. In both regions, an increasing proportion of admissions for other CHD were for coronary procedures (mainly angiogram) in Oxford (71\% in $1996 ; 84 \%$ in 2013) and WA (88\% in 1996 ; $91 \%$ in 2013).

Conclusion Since 2004, rates of all CHD subgroups have fallen in both countries, with greater declines in acute than chronic presentations. The slower declines and high proportion of chronic CHD admissions involving coronary procedures requires greater focus. Differing MI trends in younger women in both countries warrant further investigation.

\section{P61 INCIDENCE, RISK FACTORS AND PROGNOSE OF ACUTE KIDNEY INJURY IN CRITICALLY ILL PATIENTS: A PROSPECTIVE COHORT STUDY IN BRAZILIAN AMAZON}

${ }^{1}$ DMT Zanetta*, ${ }^{2}$ EA Burdmann, ${ }^{3}$ FAF Melo. ${ }^{1}$ School of Public Health, University of Sao Paulo, Sao Paulo, Brazil; ${ }^{2}$ Medical School, University of Sao Paulo, Sao Paulo, Brazil; ${ }^{3}$ Medical School, Acre Federal University, Rio Branco, Brazil

10.1136/jech-2019-SSMabstracts.212

Background Epidemiological studies, mainly prospective population-based studies, of acute kidney injury (AKI) are still scant, especially in low-income and middle-income countries ( $>85 \%$ of the world's population). This study aimed to identify incidence and factors associated AKI in critically ill patients of Brazilian Amazon, a region with limited health care facilities.

Methods Prospective cohort study of all adult patients without chronic renal disease admitted, and staying $>2$ days, in all Intensive Care Units(ICU) of Rio Branco, the largest city in Acre State, covering approximately $70 \%$ of the state population (800,000 inhabitants), from Feb 2014 to Feb 2016. Incidence, risk factors and outcomes of AKI (diagnosed by KDIGO criteria) were evaluated. Patients were followed for up to 7 days, discharge or death. Mortality was assessed $30 \mathrm{~d}$ after ICU discharge. Factors associated with AKI development and, in those with incident AKI, associated with mortality were evaluated in multiple logistic regression analysis. The proportional multiple Cox analysis evaluated 30-day mortality in non-AKI and AKI patients, using SPSS(v.22.0) software.

Results Of 1,494 patients admitted, 1,073 fulfilled selection criteria. AKI incidence was 52\% (Stage $1=62.1 \%, 2=15.6 \%$ and $3=22.2 \% ; 8.2 \%$ received dialysis). $60 \%$ of patients were admitted due to clinical condition, 25\% were hemodynamically unstable, $19 \%$ had respiratory failure. Only $2.2 \%$ had tropical diseases. Risk factors for AKI were age (adjusted OR (aOR) 1.2[CI95\% 1.1-1.3 for 10 years increase]), positive fluid balance $>1500 \mathrm{ml} / 24 \mathrm{~h}$ (aOR 2.9[2.1-3.9]), APACHE score (aOR 1.06[1.04-1.07 per unit increase]), clinical patients (reference surgical: aOR 1.6[1.2-2.6]). AKI had higher ICU mortality (AKI 43.4\% vs non-AKI 13.9\%). AKI mortality was associated with age (aOR 1.3[1.1-1.4 for 10 years increase]), mechanical ventilation (aOR 5.2[3.0-9.0]), KDIGO stage 3 (ref 1) (aOR 1.6[1.03-2.5]), vasoactive drugs or shock (aOR 2.6[1.4-4.7]), and sepsis (aOR 2.3[1.6-4.7]). Adjusted AKI hazard for 30 days after ICU discharge mortality was 1.8 (1.1-3.0).

Conclusion AKI incidence was strikingly high in critically ill patients in the Brazilian Amazon. Hospitalizations due to tropical diseases were rare, likely due to particular conditions of the Amazon area, with difficulty access to larger cities and limited health care facilities. AKI etiology and risk factors were similar to those seen in developed countries. However, mortality rates were higher. The follow-up of the cohort was for7 days and data collected may not represent all factors affecting outcomes. Poor social-economic conditions and infrastructure of health services may explain the high incidence and mortality rates for AKI observed. The results may contribute to the care of this group of patients.

\section{P62 HOW MUCH OF THE DISABILITY-RELATED INEQUALITIES IN HEALTH AND WELL-BEING ARE MEDIATED BY BARRIERS TO PARTICIPATION FACED BY PEOPLE WITH DISABILITIES? A CAUSAL MEDIATION ANALYSIS USING LONGITUDINAL DATA FROM WORKING AGE PEOPLE WITH AND WITHOUT DISABILITIES IN GREAT BRITAIN}

${ }^{1} Z$ Aitken*, 'G Disney, 'L Krnjacki, 'A Milner, ${ }^{2} E$ Emerson, ${ }^{1}$ AM Kavanagh. 'Melbourne School of Population and Global Health, The University of Melbourne, Melbourne, Australia; ${ }^{2}$ Centre for Disability Research, Faculty of Health and Medicine, Lancaster University, Lancaster, UK

\subsection{6/jech-2019-SSMabstracts.213}

Background Large health inequalities exist between people with and without disabilities, including many health conditions unrelated to the impairment causing the disability. There is some evidence that these health inequalities are, in part, due to the poor socio-economic circumstances experienced by people with disabilities, and therefore are amenable to public health intervention. In this study, we used a unique dataset to examine the relationship between disability acquisition and subsequent health outcomes using five different measures of health and wellbeing. We quantified the extent to which social barriers to participation explained the health inequalities between people with and without disability.

Methods We used data from three waves of the Life Opportunities Survey, a longitudinal study of disability in Great Britain with a strong focus on barriers to participation in society. We compared health and well-being outcomes between adults who recently acquired an impairment and those who remained disability-free, adjusting for baseline demographic, socio-economic 